\title{
Energy dissipation of a particle colliding on a flat surface
}

\author{
Fabricio Éric Fernández ${ }^{1}$, Marcelo Fabián Piva ${ }^{1}$, Román Gustavo Martino ${ }^{2}$, and María Alejandra Aguirre ${ }^{3 *}$ \\ ${ }^{1}$ Grupo de Medios Porosos, Fac. de Ingeniería, Universidad de Buenos Aires, Paseo Colón 850, (C1063ACV) Buenos Aires, Argentina. \\ ${ }^{2}$ IDIT - CONICET - Universidad Nacional de Córdoba, Av. Velez Sarsfield 1611, (5000) Córdoba, Argentina. \\ ${ }^{3}$ Grupo de Medios Porosos, Fac. de Ingeniería, Universidad de Buenos Aires, CONICET, Paseo Colón 850, (C1063ACV) Buenos \\ Aires, Argentina.
}

\begin{abstract}
To gain an understanding of the factors affecting the interaction of one grain with its environment as it reaches equilibrium, we study a particle bouncing off a flat surface. The bouncing of the particle leads to dissipation that is usually characterized with $\varepsilon$, the coefficient of restitution, defined as the ratio between the velocity component that is normal to the contact surface just before impact $\left(V_{n}\right)$ and the same component, but immediately after the collision $\left(V_{n}\right)$, i.e. related to a kinetic energy corresponding to motion in the normal direction. We will show how $\varepsilon$ is affected by energy stored in other degrees of freedom and transferred to kinetic energy that leads to an increase in normal velocity after the impact $V_{n}{ }^{\prime}$, and therefore to $\varepsilon>1$. For this purpose, the evolution of potential, translational kinetic energy and rotational kinetic energy is analysed during the whole relaxation process and just before and after each collision for two different types of particle, a disk and a faceted particle.
\end{abstract}

\section{Introduction}

This work aims to analyse energy dissipation while a grain interacts with its environment as it reaches equilibrium. This is of interest because granular systems are highly dissipative and the way they lose energy and reach equilibrium is essential to understand stability problems of granular systems, such as the triggering of avalanches (loss of stability) and their arrest (stable or metastable equilibrium) [1-4].

Usually, dissipation effects due to collisions are characterized by $\varepsilon$, the so-called coefficient of restitution that depends on various factors including material properties, body geometry and impact velocity [5-8]. This coefficient is defined as the ratio between the normal component of the relative velocity, at the contact point, before and after a collision [5-7]. If the particle collides with a surface at rest, this coefficient is the ratio between velocities components that are normal to the contact surface just before impact $\left(V_{i n}\right)$ and immediately after the collision $\left(V_{\text {out }}\right)$. It is expected $\varepsilon \leq 1$, where equality corresponds to the case of a perfectly elastic collision.

In many situations, the shape of the grains or the experimental configuration does not allow the detection and measure of the impact point and rotations. Therefore, $V_{\text {in }}$ and $V_{\text {out }}$ are usually estimated with $V_{n}$ and $V_{n}{ }^{\prime}$, the normal translational velocities of the geometrical centre of particles before and after the impact, respectively. Therefore, usually $\varepsilon$ is usually defined in the following way:

$$
\varepsilon=-\frac{V_{n}^{\prime}}{V_{n}}
$$

To analyse how values of $\varepsilon$, defined by Eq. 1, are affected by the neglected rotation and possible associated effects, e.g. tangential motion, we studied the behaviour of a particle relaxing to its resting state after successively bouncing off a flat surface. The behaviour of two very different types of particles is analysed: a disk and a faceted particle. In the case of the faceted particle, the rotation effects neglected by Eq. 1, lead to $\varepsilon>1$. This fact was analysed in terms of energy stored in other degrees of freedom and transferred to kinetic energy that leads to an increase in normal velocity after the impact $V_{n}{ }^{\prime}$. For this purpose, the transfer of energy to rotation and other degrees of freedom was studied, so that the evolution of kinetic, potential and rotation energy was analysed during the whole relaxation process and just before and after each collision.

\section{Experimental procedure}

We analysed the behaviour of a polylactide (PLA) printed faceted particle with faces that are nonagons, i.e. 9 sides polygons, and a PLA printed disk, both with a centred hole as particles used in another previous work [1]. Both particles have a thickness of $4 \mathrm{~mm}$. Other dimensions for these particles are given in Table 1. Each particle has, near the border, a small circular hole (of $2 \mathrm{~mm}$ of diameter) that allows tracing rotations with respect to their centre of mass. Despite the small hole, it should be noted that for each particle the position of its centre of mass can be assumed to coincide with its geometrical centre. Dimensions of the faceted particle were chosen so both particles share the same area $\mathrm{A}=(7.1 \pm 0.1) \mathrm{cm}^{2}$, i.e. same mass $\mathrm{m}=(2.7 \pm 0.5) \mathrm{g}$.

* Corresponding author: maaguir@fi.uba.ar

A video is available at https://doi.org/10.48448/dw75-fj74

(C) The Authors, published by EDP Sciences. This is an open access article distributed under the terms of the Creative Commons Attribution License 4.0 
The experimental procedure consists of dropping a particle inside a vertical cell. The cell is made of an aluminium frame that holds two pieces of glasses of length $\mathrm{L}=(21.5 \pm 0.1) \mathrm{cm}$ and width $\mathrm{W}=(30.0 \pm 0.1) \mathrm{cm}$ separated with a gap of $4.2 \mathrm{~mm}$, slightly larger than the depth of the particles.

Table 1. Particles dimensions and main characteristics.

\begin{tabular}{|c|c|c|}
\hline & Disk & Nonagon \\
\hline Particles & & \\
\hline $\mathrm{R}(\mathrm{cm})$ & $1.5 \pm 0.1$ & $1.6 \pm 0.1$ \\
\hline $\mathrm{L} 9(\mathrm{~cm})$ & - & $1.1 \pm 0.1$ \\
\hline $\mathrm{r}(\mathrm{cm})$ & $0.75 \pm 0.1$ & $0.75 \pm 0.1$ \\
\hline
\end{tabular}

The particle centre is aligned with the upper border of the cell, i.e. at a distance $H_{o}=(21.5 \pm 0.1) \mathrm{cm}$ to the base, and it is released, without spin, from this height $\mathrm{H}_{\mathrm{o}}$. The particle collides with the flat bottom surface, consisting of a plexiglass piece of $5 \mathrm{~cm}$ of thickness. The particle successively bounces until it relaxes and stops moving.

The whole process is registered by a Mako U-051B camera at a sampling rate of $391 \mathrm{fps}$ with a pixel resolution of $800 \times 600$. The observation window is 26.4 $\mathrm{cm} \times 19.8 \mathrm{~cm}$. Thus, 1 pixel is equivalent to $0.033 \mathrm{~cm}$ in the physical space. The aluminium frame of the cell does not allow to register the launching point, so the first registered values for the vertical coordinate of the centre of mass is $\mathrm{Y}_{\mathrm{CM}} \approx 16 \mathrm{~cm}$.

Image processing with ImageJ [9] allowed to determine the positions of the centre of mass and of the circular mark previously mentioned, allowing the measurement of trajectories and translational and rotational velocities. A sudden change in the sign of the vertical velocity occurs when the particle collides with the flat surface and therefore $V_{n}$ and $V_{n}{ }^{\prime}$ can be obtained respectively from the local minima and maxima of the evolution of the vertical velocity (Fig. 1). Considering the sampling rate and that a minimum displacement of 1 pixel can be detected, values for $V_{n}$ and $V_{n}{ }^{\prime}$ less than $13 \mathrm{~cm} / \mathrm{s}$ could not be accurately measured and are filtered from measured data. For each rebound, a value of $\varepsilon$, the coefficient of restitution was obtained with $V_{n}$ and $V_{n}{ }^{\prime}$ (Eq. (1)) estimated with translational velocities of the geometrical centre of particles before and after the impact, respectively. Considering the size of the particles and that thresholds used in image analyses are of approximately 200, coordinates are obtained with uncertainties of 0.1 pixels [10]. Therefore, velocity values have an error of $\Delta \mathrm{V}=2.6 \mathrm{~cm} / \mathrm{s}$ which leads to relative errors $\Delta \varepsilon / \varepsilon \in[0.03,0.19]$. For $\varepsilon \leq 1$, error $\Delta \varepsilon \in[0.02,0.19]$ and for $\varepsilon>1$, error $\Delta \varepsilon \in[0.04,0.33]$.

Also, we obtain the potential energy, translational kinetic energy and rotational kinetic energy, which are analysed during the relaxation process. It should be noted, that in successive bounces, after the first one, the face with which the faceted particle impacts cannot be controlled, we consider that a significant change in the results will not be introduced by controlling the launching angle of the face.

\section{Experimental results}

In this section, we report experimental results for 412 and 1296 collisions of the disk and faceted particle, respectively.

\subsection{Evolution of vertical and horizontal coordinates}

Figure 1 displays the typical evolution of vertical and horizontal coordinates observed for each particle. In any case, the particle falls from a height $\mathrm{H}_{n}$, after a collision the particle rebounds and reaches a maximum height $\mathrm{H}_{\mathrm{n}+1}<\mathrm{H}_{\mathrm{n}}$, which indicates that, as expected, energy has been lost in the process. Disk motion is always in the vertical direction, different from the faceted particle that might present a lateral displacement (inset on the bottom panel of Fig. 1) if a vertex collides with the flat bottom surface. Also, the evolution of the velocity in the vertical direction is shown in Fig. 1 where local minima and maximum correspond to $V_{n}$ and $V_{n}{ }^{\prime}$, respectively.
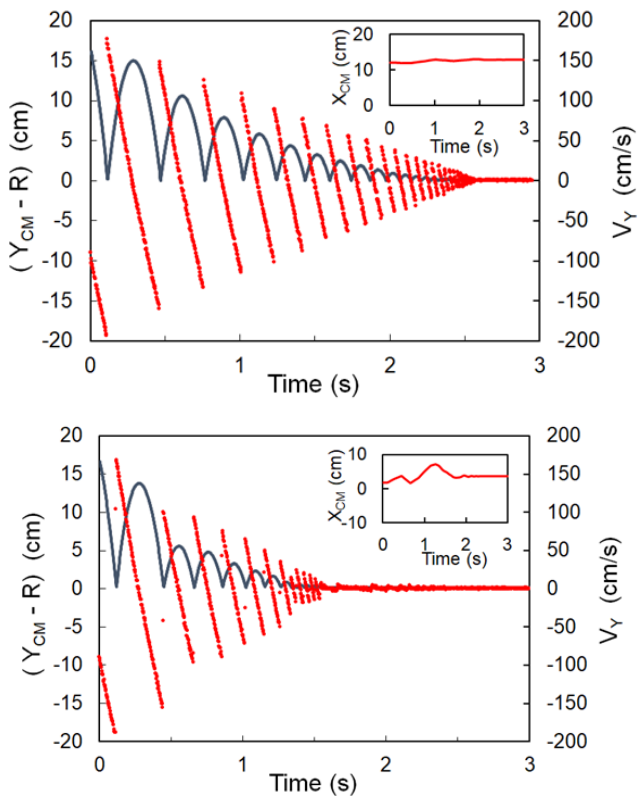

Fig. 1. Example of the evolution of the vertical coordinate of the centre of mass (left axis) and velocity (right axis) while the particle relaxes to equilibrium after successive bounces. Inset: Evolution of the horizontal coordinate of the centre of mass. Top panel and inserted figure: disk. Bottom panel and inserted figure: faceted particle.

\subsection{Coefficient of restitution}

Energy dissipation is characterized with $\varepsilon$, the coefficient of restitution (Eq. 1) that does not account for effects due to rotations. We present results for $\varepsilon$ as a function of the normal impact velocity for the disk and the faceted particle (Fig. 2). 
As reported in others works [5-7], we observed that the dispersion of $\varepsilon$ increases as the impact velocity decreases (insets in Fig. 2). It is worth noting that dispersion is considerably larger for the faceted particle and that for small $V_{n}$ values the distribution of $\varepsilon$ is asymmetric around each mean value. Later, we will propose a preliminary explanation in terms of energy transfer.

Draws attention the fact that, for the faceted particle, $\varepsilon$ might be larger than 1 for small impact velocities (bottom panel in Fig. 2). We observed that $9.72 \%$ of the collisions have $\varepsilon>1$. This fact might be understood in terms of energy stored in other degrees of freedom, during a collision, and transferred, in a later impact, to kinetic energy that leads to an increase in normal velocity after the impact $V_{n}{ }^{\prime}$. The increase of energy in some degrees of freedom, at impact, and its release at another collision will be shown in the following subsection.
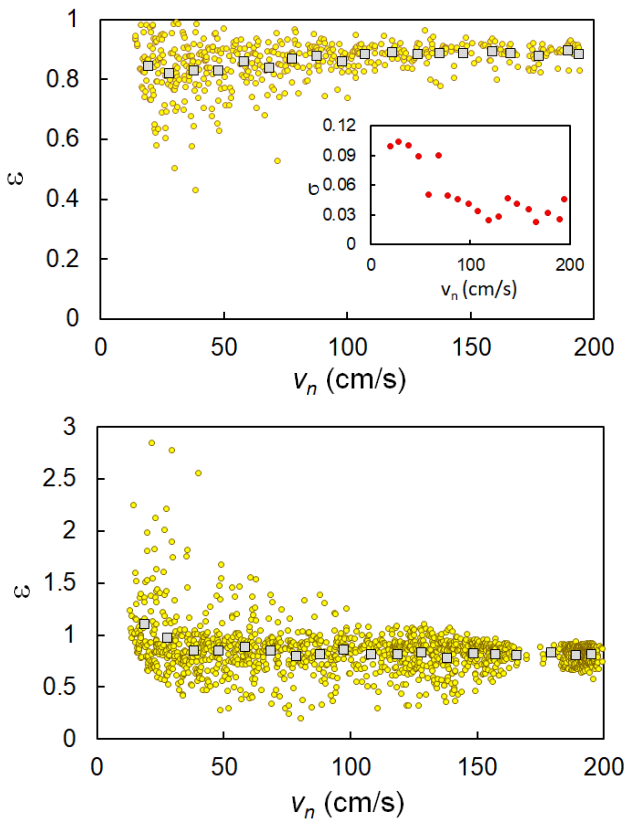

Fig. 2. Coefficient of restitution as a function of $V_{n}$, the normal (vertical) impact velocity for a particle colliding with a flat surface. Square markers are mean values of $\varepsilon$ sampled in windows of width $10 \mathrm{~cm} / \mathrm{s}$ for $V_{n}$, plotted vs. $\left\langle V_{n}\right\rangle$ obtained in the mentioned windows. Inset: shows standard deviation for values of $\varepsilon$ sampled in windows of width $10 \mathrm{~cm} / \mathrm{s}$ for $V_{n}$. Top panel: disk. Bottom panel: faceted particle.

In figure 3 we present mean values of $\varepsilon$, with uncertainty bars estimated with its standard deviations, as a function of mean values of $\left\langle V_{n}\right\rangle$. Both $\langle\varepsilon\rangle$ and $\left\langle V_{n}\right\rangle$. are calculated for samples obtained in windows of width $10 \mathrm{~cm} / \mathrm{s}$ for $V_{n}$.

On the one hand, as $\left\langle V_{n}>\right.$ decreases, faceted particles increase $<_{\varepsilon}>$ above values of obtained for disks and reaches values larger than 1 . On the other hand, for $\left\langle V_{n}>>(70 \pm 1) \mathrm{cm} / \mathrm{s}\right.$, values of $\langle\varepsilon>$ for disks are larger than those obtained for the faceted particle. Considering only collisions where $\varepsilon \leq 1$, mean values for the coefficient of restitution, for disks and faceted particle, are respectively: $\left\langle_{\varepsilon}>^{\text {disk }}=0.87 \pm 0.06\right.$ and $<_{\varepsilon}>^{\text {faceted }}=0.75 \pm 0.05$, indicating that on average faceted particle dissipate more energy at collisions except when $V_{n}$ are lower than approximately $40 \mathrm{~cm} / \mathrm{s}$.

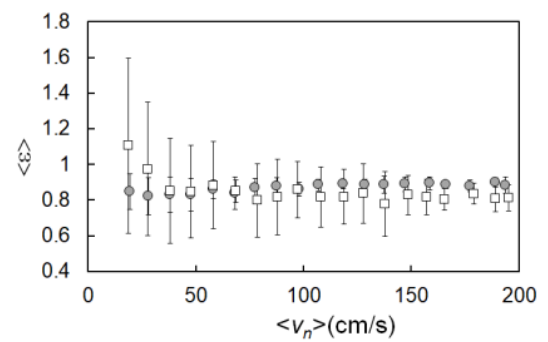

Fig. 3. Mean values $\left\langle_{\varepsilon}\right\rangle$ and its standard deviations $\sigma$ obtained in windows of width $10 \mathrm{~cm} / \mathrm{s}$ for $V_{n}$. (Disks with filled circles and faceted particle with empty squares). Magnitudes are plotted vs. mean values $\left\langle V_{n}\right\rangle$ obtained in the mentioned windows.

\subsection{Energy evolution during the relaxation process}

Initially, a particle is dropped from a height $\mathrm{H}_{\mathrm{o}}$ with zero velocity and spin. During the fall, the particle (disk or faceted particle) only moves in the vertical direction and does not rotate. After colliding with the flat bottom, disk and the faceted particle present different behaviours:

- Disk: during a collision, no torques appear because the centre of mass and the point of impact are aligned with gravity (vertical direction) and the disk has a homogeneous mass distribution with respect its centre of mass. Therefore, $E_{K}$, translational kinetic energy only accounts for motion in the vertical direction $\left(\mathrm{E}_{\mathrm{K}}\left(\mathrm{V}_{\mathrm{y}}\right)\right)$ and $E_{R}$, rotational kinetic energy is zero during the whole relaxation process. An example is shown in Fig. 4 (top panel).

- Faceted particles: if a vertex of the faceted particle collides with the flat bottom, a torque might be induced leading to rotations and/or lateral displacement (inset of the bottom panel in Fig. 1) when the impact point and the centre of mass are not aligned with gravity (vertical direction). Therefore, $E_{K}$, translational kinetic energy accounts for motion in both, vertical and horizontal, directions $\left(E_{K}\left(V_{x}, V_{y}\right)\right)$ and $E_{R}$, rotational kinetic energy might be larger than zero and remain constant between two consecutive collisions. Afterwards, the previous gained translational kinetic energy associated to horizontal motion and/or rotational kinetic energy can either be lost at impact or can be, in part, transfer to translational kinetic energy associated to vertical motion accounting for an increase of $V_{n}{ }^{\prime}$ a later impact. For example, in the bottom panel in Fig. 4, the second collision induces a rotation, energy is transfer to the rotational kinetic energy where it is stored until, in the third rebound, it is released.

Values of $\varepsilon$ were obtained with the limitations specified for Eq. 1 (Sec. 1), therefore, to understand energy transfers leading to collisions with $\varepsilon>1$ for faceted particles, we analyse variations, before and after a collision, of translational kinetic energy in the vertical direction $\Delta \mathrm{E}_{\mathrm{K}}\left(\mathrm{V}_{\mathrm{y}}\right)$ due to variations of rotational kinetic $\Delta \mathrm{E}_{\mathrm{R}}$, translational kinetic associated to horizontal direction $\Delta \mathrm{E}_{\mathrm{K}}\left(\mathrm{V}_{\mathrm{x}}\right)$ and potential energy $\Delta \mathrm{E}_{\mathrm{p}}$. It should be noted that $\Delta \mathrm{E}_{\mathrm{p}}$ should be considered because the sampling rate leads to a difference in the height for 
positions of the particle where magnitudes just before and after each collision is calculated.

For $\varepsilon>1$ we obtained the following ratios: $\Delta \mathrm{E}_{\mathrm{K}}\left(\mathrm{V}_{\mathrm{y}}\right) / \Delta \mathrm{E}_{\mathrm{R}}, \Delta \mathrm{E}_{\mathrm{K}}\left(\mathrm{V}_{\mathrm{y}}\right) / \Delta \mathrm{E}_{\mathrm{K}}\left(\mathrm{V}_{\mathrm{x}}\right)$ and $\Delta \mathrm{E}_{\mathrm{K}}\left(\mathrm{V}_{\mathrm{y}}\right) / \Delta \mathrm{E}_{\mathrm{P}}$. When $\varepsilon>1$, there is an increase of $\mathrm{E}_{\mathrm{K}}\left(\mathrm{V}_{\mathrm{y}}\right)$ after the collision so $\Delta \mathrm{E}_{\mathrm{K}}\left(\mathrm{V}_{\mathrm{y}}\right)>0$. This increment should be explained with loss or energy transfer from $\mathrm{E}_{\mathrm{R}}, \mathrm{E}_{\mathrm{K}}\left(\mathrm{V}_{\mathrm{x}}\right)$ and $\mathrm{E}_{\mathrm{P}}$ to $\mathrm{E}_{\mathrm{K}}\left(\mathrm{V}_{\mathrm{y}}\right)$, i.e. $\Delta \mathrm{E}_{\mathrm{R}}<0, \Delta \mathrm{E}_{\mathrm{K}}\left(\mathrm{V}_{\mathrm{x}}\right)<0$ or $\Delta \mathrm{E}_{\mathrm{P}}<0$, leading to ratios smaller than zero. Therefore, we will analyse collisions with ratios less than zero. As mentioned, $9.72 \%$ of the collisions (126 collisions) have $\varepsilon>1$. We observed that:

- Few collisions with $\varepsilon>1$ (18\%) show a possible transfer of $E_{K}\left(V_{x}\right)$ to $E_{K}\left(V_{y}\right)$. Moreover, in $60 \%$ of those impacts, the amount of energy that might be transferred is very small and cannot account for the observed $\Delta \mathrm{E}_{\mathrm{K}}\left(\mathrm{V}_{\mathrm{y}}\right)$ values $\left(\Delta \mathrm{E}_{\mathrm{K}}\left(\mathrm{V}_{\mathrm{y}}\right)>\left|\Delta \mathrm{E}_{\mathrm{K}}\left(\mathrm{V}_{\mathrm{x}}\right)\right|\right)$.

- Most collisions with $\varepsilon>1$ (94\%) show a possible transfer of $\mathrm{E}_{\mathrm{P}}$ to $\mathrm{E}_{\mathrm{K}}\left(\mathrm{V}_{\mathrm{y}}\right)$ but in $68 \%$ of them, the amount of energy that might be transfer is very small and cannot account for the observed $\Delta \mathrm{E}_{\mathrm{K}}\left(\mathrm{V}_{\mathrm{y}}\right)$ values $\left(\Delta \mathrm{E}_{\mathrm{K}}\left(\mathrm{V}_{\mathrm{y}}\right)>\left|\Delta \mathrm{E}_{\mathrm{P}}\right|\right)$.

- Most collisions with $\varepsilon>1$ (99\%) show a possible transfer of $E_{R}$ to $E_{K}\left(V_{y}\right)$ and of those, $99.4 \%$ present variations that are large enough to account for the observed $\Delta \mathrm{E}_{\mathrm{K}}\left(\mathrm{V}_{\mathrm{y}}\right)$ values $\left(\mathrm{E}_{\mathrm{K}}\left(\mathrm{V}_{\mathrm{y}}\right) \leq\left|\Delta \mathrm{E}_{\mathrm{R}}\right|\right)$, which indicates that energy stored as rotational kinetic energy is effectively increasing $V_{n}{ }^{\prime}$ leading to $\varepsilon>1$, with the limitations specified for Eq. 1 .
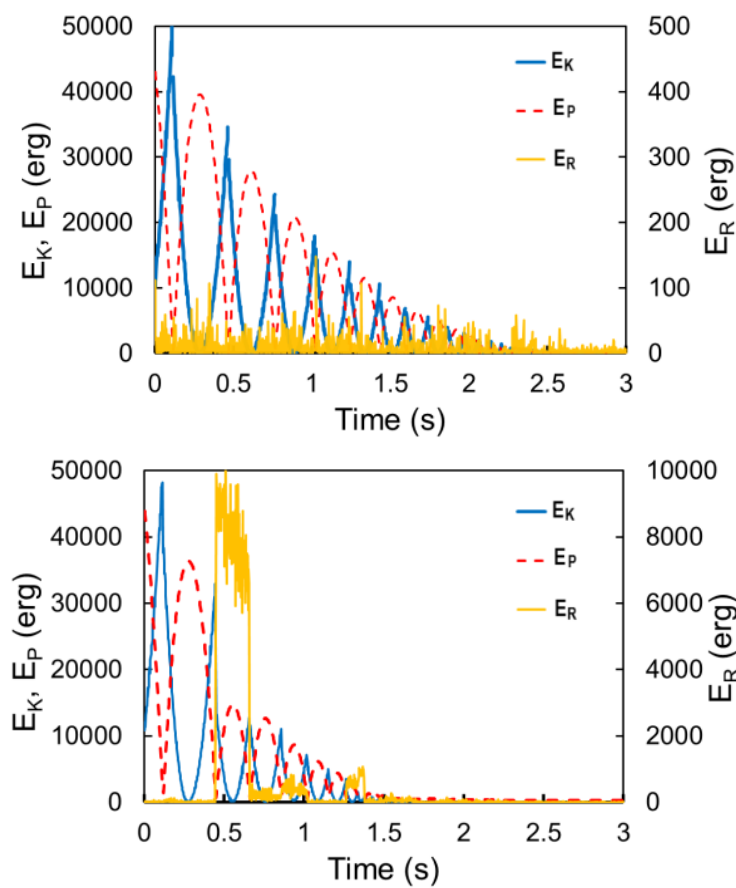

Fig. 4. Example of energy evolution during the relaxation process of a particle: translational kinetic energy (blue solid line), rotational kinetic energy (yellow solid line) and potential energy (red dashed line). Top panel: disk. Bottom panel: faceted particle.

To analyse which type of energy is most likely to be transferred and stored as rotational kinetic energy, a similar analysis was carried on for collisions with $\varepsilon<1$, and we observed that $70 \%$ of collisions might generate a transfer of $E_{K}\left(V_{y}\right)$ to $E_{R}: \Delta E_{R}<\left|\Delta E_{K}\left(V_{y}\right)\right|$.

\section{Summary and conclusions}

The coefficient of restitution is generally used as a measure to characterize the dissipation that occurs in the collisions that determine the behaviour of granular systems and is measured with limitations specified for Eq. 1 in Sec. 1. In this work, we observed that:

- as noticed by other researchers [5-7], $\varepsilon$ slightly depends on the impact speed $V_{n}$ and presents a greater variation for low values of $V_{n}$ and is even higher for the case of the faceted particle;

- at low impact speeds, $\varepsilon$ can take values greater than 1 , for case of the faceted particle;

- in those experiments, the appearance of torques can induce rotations and lateral motion leading to the storage of rotational and translational energy in directions other than normal (vertical).

- we observed that the latter fact is due to collisions with $\varepsilon<1$ that transfer $\mathrm{E}_{\mathrm{K}}\left(\mathrm{V}_{\mathrm{y}}\right)$ mainly to $\mathrm{E}_{\mathrm{R}}$, then the rotational mode stores energy until, during an impact, part is transfer mostly back to $\mathrm{E}_{\mathrm{K}}\left(\mathrm{V}_{\mathrm{y}}\right)$ leading to collisions with $\varepsilon>1$.

So, we conclude that the following factors should be considered to evaluate if it is pertinent only to use $\varepsilon$ (Eq. 1) as a parameter to characterize impact dissipation with the limitations specified in Sec. 1:

1) the presence of edges and roughness both in the particle and on the surface, which might induce torques and therefore rotations;

2) the range of possible impact speeds that also condition the value of this coefficient.

This work has been supported by the ANPCyT (Argentina) through Grant No. PICT-2014-2587, and the Centro Argentino Francés de Ciencias de la Ingeniería (CAFCI, CONICETCNRS) through Grant No. R147-15.

\section{References}

[1] M. Piva, R.G. Martino, J.-C. Géminard, M.A. Aguirre, Phys. Rev. E 98, 062902 (2018)

[2] R. Fischer, P. Gondret, B. Perrin, M. Rabaud, Phys. Rev. E 78, 021302 (2008)

[3] C.J. Olson, C. Reichhardt, M. McCloskey, R.J. Zieve, Europhys. Lett. 57, 904 (2002)

[4] F. Cantelaube, Y. Limon-Duparcmeur, D. Bideau, G.H. Ristow, J. Phys. (France) I 5, 581 (1995)

[5] M. Montaine, M. Heckel, C. Kruelle, T. Schwager, T. Pöschel, Phys. Rev. E 84, 041306 (2011)

[6] H. King, R. White, I. Maxwell, N. Menon, Europhys. Lett. 93, 14002 (2011)

[7] R. Sondergaard, K. Chaney, C.E. Brennen, J. Appl. Mech. 57, 694 (1990)

[8] P. Gondret, M. Lance, L.Petit, Phys. Fluids, 14, 643, (2002)

[9] C.A. Schneider, W. S. Rasband, K.W. Eliceiri, Nat. Methods 9, 671 (2012)

[10] Y. Feng, J. Goree, Bin Liu, Rev. Sci. Instrum. 78, 053704 (2007) 